\title{
Equipamento brasileiro de detecção gama intra-operatória para biópsia de linfonodo sentinela
}

\section{Brazilian gamma detection device for sentinel lymph node biopsy}

Renato Santos de Oliveira Filho, TCBC-SP1; Andréla Bufoni Farah ${ }^{2}$; Fábio Eduardo da Costa ${ }^{3}$; Fabio Xerfan Nahas, TCBC-SP4; Daniel Arcuschin de Oliveira ${ }^{5}$; Renan Gianotto de Oliveira ${ }^{5}$

\section{R E S U M O}

\begin{abstract}
Objetivo: Testar a eficácia de equipamento de detecção gama intra-operatória (DGI) desenvolvido pelo IPEN (Brasil), em procedimentos de biópsia de linfonodo sentinela (BLS) no melanoma e no câncer de mama. Métodos: Foram estudados 40 pacientes portadores de melanoma ou câncer de mama com indicação para realização de BLS.Todos pacientes foram submetidos à linfocintilografia e a BLS ocorreu entre 2 a 24 horas após a mesma. Concomitantemente à DGI, realizou-se o mapeamento linfático com corante vital. Foram feitas leituras com o equipamento convencional Neoprobe ${ }^{\circledR} 1500$ e com o equipamento em teste (IPEN) dos valores de captação do sítio de injeção do radiofármaco, do LS in vivo e ex vivo e da captação de fundo. Foi registrado se o LS estava corado e se o cirurgião teve facilidade para encontrá-lo. Nos primeiros 20 pacientes utilizou-se o equipamento convencional e depois o de teste; nos outros 20, utilizou-se primeiro o equipamento em teste, com objetivo de verificar se o mesmo identificava primariamente o LS. Resultados: Dos quarenta pacientes, 33 eram portadores de tumor de mama e sete de melanoma cutâneo; variação da idade: 21 a 68 anos (mediana= 46 anos); 35 mulheres e 5 homens. Em apenas um paciente o LS não foi encontrado, nem pela DGI nem pelo corante vital. Não houve diferença estatística entre as razões ex vivo/fundo obtidas com os dois equipamentos ( $p=0,2583-n s)$. Conclusão: É possível realizar o procedimento de BLS com o equipamento brasileiro desenvolvido pelo IPEN, com facilidade e sem prejuízo para o paciente.
\end{abstract}

Descritores: Biópsia de linfonodo sentinela; Raios gama; Melanoma; Mama.

\section{INTRODUÇÃO}

A utilização da detecção gama intra-operátoria (DGI) já é estabelecida em oncologia para identificação de linfonodos sentinelas em melanoma e em câncer de mama. Sua aplicação vem sendo estudada para outros tumores como os de vulva e os de cabeça e pescoço $0^{1,2}$. É considerada hoje essencial e indispensável para o procedimento de biópsia do linfonodo sentinela (BLS). Apresentando alta sensibilidade para detecção dos linfonodos, a DGI permite menor dissecção, tornando o procedimento ainda menos invasivo. A BLS tem diminuído o número de linfadenectomias radicais desnecessárias e, conseqüentemente reduzindo a morbidade cirúrgica ${ }^{3}$.

O linfonodo sentinela (LS) é definido como o primeiro linfonodo da base linfonodal para a qual ocorre a drenagem do tumor primário. O estado histopatológico do LS prediz com acurácia o status da base linfática regional. A BLS é um procedimento diagnóstico que possibilita a identificação de micrometástases numa fase em que ainda não podem ser detectadas por exames de imagem ${ }^{4,5}$.

O procedimento de BLS envolve a linfocintilografia pré-operatória, o mapeamento linfático intra-operatório com corante vital, a DGI e o exame histopatológico do LS $6-8$.

A DGI complementa o mapeamento linfático com corante vital, facilitando a correta identificação do LS, diminuindo o tempo cirúrgico e permitindo menor incisão. Procura-se o linfonodo de maior captação, podendo este estar ou não corado. Atualmente, existem vários equipamentos disponíveis comercialmente para a realização da DGI (Neoprobe ${ }^{\circledR}$, Europrobe ${ }^{\circledR}$, Navigator ${ }^{\circledR}$, etc.). Os aparelhos disponíveis são basicamente constituídos por uma sonda (probe) em cuja extremidade distal está o cristal ou pequena gama-câmara, a qual é acoplada a um contador portátil. A utilização de colimadores opcionais acompanha alguns modelos e seu uso fica a critério do cirurgião.

No Brasil, os equipamentos disponíveis são importados e de alto custo, o que muitas vezes limita o seu uso, restringindo-se aos grandes centros. O IPEN desenvolveu um equipamento de DGI de sonda cirúrgica radioguiada912(figura1). Pretendemos com este estudo testar a eficácia deste aparelho, em procedimentos de BLS em humanos, comparando-o com um equipamento importado convencional (Neoprobe 1500®).

Trabalho realizado no Hospital Israelita Albert Einstein, São Paulo- SP - Brasil.

1. Cirurgião Oncológico do Hospital Israelita Albert Einstein - São Paulo -SP-BR. 2. Cirurgiã Plástica do Hospital Israelita Albert Einstein - São Paulo -SP-BR. 3. Doutor em Ciências na Área de Tecnologia Nuclear, IPEN - São Paulo -SP-BR. 4. Cirurgião Plástico da Universidade Federal de São Paulo - São Paulo -SP-BR. 5. Acadêmico de Medicina da Faculdade de Medicina Anhembi-Morumbi - São Paulo -SP-BR. 


\section{MÉTODOS}

Após aprovação do Comitê de Ética em Pesquisa do Instituto de Pesquisa do Hospital Israelita Albert Einstein iniciou-se estudo prospectivo com pacientes portadores de melanoma ou câncer de mama com indicação para realização de biópsia de linfonodo sentinela. Foram incluídos pacientes que concordaram com o estudo e que assinaram o termo de consentimento pós-informado, no período de julho de 2007 a dezembro de 2008.

Todos os pacientes foram submetidos a linfocintilografia pré-operatória com o isótopo radioativo ${ }^{99}$ tecnécio (emissor gama em $140 \mathrm{keV}$ ) ligado ao dextran 500 ou fitato, definindo a região linfática de drenagem e LS, com marcação na pele do local de maior captação. A BLS ocorreu de 2 a 24 horas após a linfocintilografia.

Conjuntamente com a detecção gama intra-operatória, realizou-se o mapeamento linfático intra-operatório com azul patente $V$ (Laboratóro Guerbet, França). A DGI foi realizada com o equipamento convencional Neoprobe $₫ 1500$, (Neoprobe Corporation, Ohio, USA) com sonda de telureto de cádmio e zinco, faixas de energia entre 10 e $180 \mathrm{keV}$, com $10 \mathrm{~mm}$ de diâmetro, e com o equipamento em teste (DGI IPEN, São Paulo, Brasil) com sonda de iodeto de césio dopada com tálio, CsI (TI), indicada para detecção de raios gama na faixa de energia de 120 a $662 \mathrm{keV}$, e com $11 \mathrm{~mm}$ de diâmetro (Figura 1).

Através das leituras das escalas numéricas e orientação sonora, o operador era guiado até a posição dos linfonodos sentinelas concentrantes, localizando-os para exérese. Para considerar um linfonodo como sentinela ele deveria apresentar pelo menos 10 vezes mais atividade que a leitura de fundo. Após exérese do LS, o leito operatório fora novamente rastreado pelo detector em busca de mais linfonodos concentrantes.

Foram feitas leituras com os dois equipamentos seqüencialmente dos valores de captação do sítio de injeção do radiofármaco, do LS in vivo (através da pele) e ex vivo bem como da captação de fundo (na face lateral da coxa contra - lateral ao tumor). Foi também registrado se o LS apresentava-se corado ou não e se o cirurgião teve facilidade ou dificuldade para encontrar o LS. A facilidade e dificuldade de encontro do LS foram avaliadas pelo tempo decorrido entre o início da incisão até o encontro do LS (= a 20 minutos ou $>20$ minutos, respectivamente). Os equipamentos foram utilizados desconectados da rede elétrica, funcionando com a bateria interna, diminuindo possíveis riscos de choque elétrico ou interferências com outras fontes de radiação eletromagnéticas.

Nos primeiros 20 pacientes utilizou-se primeiramente o equipamento convencional e depois o equipamento de teste em cada leitura de cada paciente. Nos outros 20 pacientes, utilizou-se primeiro o equipamento em teste, com objetivo de verificar se o mesmo identificava primariamente o LS.

\section{RESULTADOS}

Dos quarenta pacientes submetidos a BLS, 33 eram portadores de tumor de mama e sete de melanoma cutâneo. A idade variou de 21 a 68 anos (mediana $=46$ anos), sendo 35 mulheres e cinco homens.

Em apenas um paciente o LS não foi encontrado, nem pela DGI nem pelo corante vital. Em todos os casos houve facilidade de encontro do LS pelo cirurgião tanto com o Neoprobe quanto com o DGI IPEN.

Os valores médios de leitura de fundo, do LS "in vivo", do LS "ex vivo" e do sítio de injeção dos dois equipamentos nos 39 pacientes podem ser vistos na tabela 1 .

Os valores das medidas das leituras de Fundo e as do LS "ex vivo" não apresentaram homogeneidade de variâncias e por este motivo foram analisados pelo teste

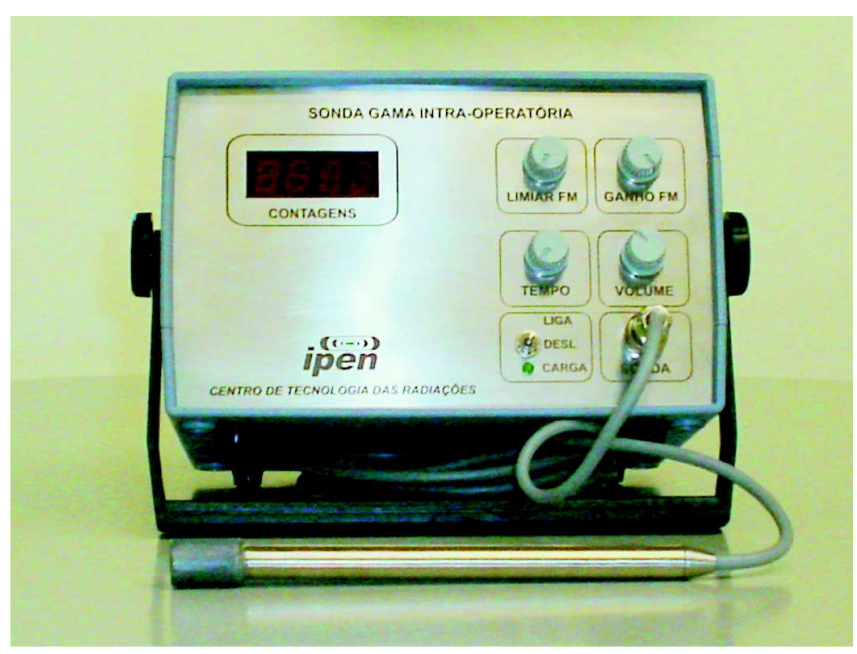

Figura 1 - Equipamento brasileiro de detecção gama intra-operatória desenvolvido pelo IPEN (São Paulo, Brasil).

Tabela 1 - Valores médios de captação de radiação gama (39 pacientes).

\begin{tabular}{|c|c|c|c|}
\hline & $\begin{array}{c}\text { Neoprobe } 1500 \\
\text { Média (mínimo - máximo) }\end{array}$ & $\begin{array}{c}\text { DGI - IPEN } \\
\text { Média (mínimo - máximo) }\end{array}$ & Teste * \\
\hline Sítio de injeção & $9120(7210-10830)$ & $7980(6420-9670)$ & \\
\hline Fundo & $25 \quad(7-\quad 31)$ & $16 \quad(12-\quad 22)$ & $T=40,5-p=0,0000011$ \\
\hline LS "in vivo" & $3530(2720-4350)$ & $2860(2240-3420)$ & \\
\hline LS "ex vivo" & $4249(3430-4920)$ & $2477(1735-3565)$ & $T=0,00-p=000000053$ \\
\hline Média das Razões & & & \\
\hline LS "ex vivo"/ Fundo & $181,99(08,67-533,33)$ & $158,88(85-$ & $\mathrm{T}=309,0-p=0,2583-n s$ \\
\hline
\end{tabular}


não-paramétrico de Wilcoxon. Este teste mostrou diferença estatisticamente significativa entre os resultados encontrados pelo Neoprobe 1500 o DGI-IPEN (apresentam escalas de medições diferentes). Entretanto, a razão LS "ex vivo" / Fundo não mostrou diferença entre os dois aparelhos ( $p=0,2583$-ns).

Nos últimos 20 pacientes, o equipamento em teste fora utilizado antes do convencional e identificou o LS corretamente em todos os casos, o que foi confirmado pelo equipamento convencional. Em todos os pacientes, exceto em dois, o LS além de hipercaptante também estava corado pelo azul patente $\mathrm{V}$.

\section{DISCUSSÃO}

O cristal cintilador de iodeto de césio dopado com tálio CsI(TI) $)^{b}$ crescido no IPEN/CNEN (Instituto de Pesquisa Energéticas e Nucleares/ Comissão Nacional de Energia Nuclear) apresenta as mesmas características dos cristais disponíveis no mercado mundial. A sua utilização em sondas radioguiadas atende às necessidades de nacionalização e redução de custos. Paralelamente, o acoplamento deste cristal a fotodiodos comerciais reduz o custo das sondas e permite fácil manutenção ${ }^{12}$.

Dentre as sondas desenvolvidas utilizou-se também o cristal semicondutor de brometo de tálio $(\mathrm{T} \mid \mathrm{Br})^{11,13}$ como novo meio detector em substituição ao cristal de telureto de cádmio (CdTe) ou telureto de cádmio e zinco (CZT). A utilização desta nova tecnologia de detectores além de mostrar a viabilidade deste cristal semicondutor nesta aplicação, cria uma independência tecnológica nacional na área de detectores de radiação para o espectro de energia coberto por este tipo de detector ${ }^{12}$. Esta indepen- dência está centrada também no crescimento destes cristais, que poucos países detêm e que tem sido crescidos e testados ${ }^{11,13} \mathrm{com}$ bons resultados pelos laboratórios do IPEN/ CNEN-SP. Os cristais de $\mathrm{TIBr}$ por serem detectores semicondutores não necessitam ser acoplados a fotodiodos, dispensando a aquisição deste componente que embora comercialmente encontrado, não deixa de ser importado e com custo substancial.

Mostrou-se que é possível realizar o procedimento de BLS com o equipamento desenvolvido pelo IPEN, sem prejuízo para o paciente. O LS foi encontrado com facilidade pelos dois equipamentos. Houve um discernimento de leitura entre o Fundo e o LS em cada paciente e uma correspondência nas razões de leitura do LS ex vivo / Fundo dos dois equipamentos, com identificação do linfonodo de maior captação radioativa. A análise estatística com o teste não-paramétrico de Wilcoxon não mostrou diferença estatisticamente significativa entre os valores da razão LS "ex vivo" / Fundo ( $p=0,2583$-ns) obtida com os dois equipamentos.

Os resultados favorecem a utilização do equipamento de detecção gama intra-operatória desenvolvido pelo IPEN, São Paulo, Brasil, para a realização de procedimentos de biópsia de linfonodo sentinela em câncer de mama e em melanoma. Este aparelho já havia sido testado experimentalmente ${ }^{14}$. A disponibilização mais econômica deste equipamento tornará mais viável a realização deste procedimento em todo o território brasileiro.

\section{Agradecimentos}

Agradecemos aos colegas mastologistas Dr.André Aguiar do Monte pelas pacientes encaminhadas.

\title{
A B S T R A C T
}

\begin{abstract}
Objective: To test the effectiveness of an intra-operative gamma detection Brazilian device (IPEN) on sentinel lymph node biopsy (SLNB) procedures. Methods: Forty melanoma or breast cancer patients with indication for undergoing SLNB were studied. Lymphoscintigraphy was done 2 to 24 hours prior to surgery. Lymphatic mapping with vital dye and gamma detection were performed intraoperatively. For gamma detection Neoprobe $\Theta 1500$ was used followed by IPEN (equipment under test) in the first 20 patients and for the remaining half IPEN was used first to verify its ability to locate the sentinel node (SN). Measurements were taken from the radiopharmaceutical product injection site, from SN (in vivo and ex vivo) and from background. It was recorded if the $S N$ was stained or not and if it was found easily by surgeon. Results: There were 33 (82.5\%) breast cancer and 7 (17.5\%) melanoma patients. Ages varied from 21 to 68 year-old (median age of 46). Sex distribution was 35 (87.5\%) women and 5 (12.5\%) men. Sentinel node was found in all but one patient. There was no statistical difference between the reasons ex vivo / background obtained with the measures of both equipments ( $p=0,2583-n s)$. The SN was easily found by the surgeon with both devices. Conclusion: The SLNB was successfully performed using either equipment. It was possible to do SLNB with the Brazilian device developed by IPEN without prejudice for the patient.
\end{abstract}

Key words: Sentinel Lymph Node Biopsy; Gamma Rays; Melanoma; Breast.

\section{REFERENCIAS}

1. van Akkooi AC, de Wilt JH, Verhoef C, Eggermont AM. Cutaneous melanoma and sentinel lymph node bio. Ann Surg Oncol. 2008; 15(6):1808-9. Epub 2008 Feb 8.

2. Faries $M B$, Morton DL. Surgery and sentinel lymph node. Semin Oncol. 2007; 34(6):498-508.
3. Amersi F, Morton DL. The role of sentinel lymph node biopsy in the management of melanoma. Adv Surg. 2007; 41:24156.

4. Essner R. Sentinel lymph node biopsy and melanoma biology. Clin Cancer Res. 2006;12(7 Pt 2):2320s-5s.

5. Oliveira-Filho RS, Ferreira LM, Barbieri $A$, et al. Linfadenectomia seletiva com biópsia de linfonodo sentinela no melanoma cutâneo. 
Experiência brasileira - Projeto FAPESP 97/02516-0. An Bras Dermatol. 2000;75:573-80.

6. Ross MI, Reintgen D, Balch CM. Selective lymphadenectomy: emerging role for lymphatic mapping and sentinel node biopsy in the management of early stage melanoma. Sem Surg Oncol. 1993; 9(3):219-23.

7. Silva AM, Oliveira-Filho RS, Ferreira LM, Saconato H. Relevance of micrometastases detected by reverse transcripatase-polymerase chain reaction for melanoma recurrence: systematic review and meta-analysis. São Paulo Med J. 2003;121(1):24-7.

8. Morton DL, Thompson JF, Essner R, Elashoff R, Stern SL, Nieweg $\mathrm{OE}$ et al. Validation of the accuracy of intraoperative lymphatic mapping and sentinel lymphadenectomy for early-stage melanoma: a multicenter trial. Multicenter Selective Lymphadenectomy Trial Group. Ann Surg. 1999; 230(4):453-63; discussion 463-5.

9. Costa FE, Rela PR, Oliveira IB, et al. Desenvolvimento de uma sonda radioguiada para mapeamento linfático intra-operatória. In: IX Brazilian Congress of Medical Physics, 2004, Rio de Janeiro. Proceedings III Iberian American and Caribbean Regional Congress of Medical Physics, 2004.

10. Costa FE, Rela PR, Pereira MCC, et al. Surgical gamma probe with CsI(TI) scintillator crystal coupled to photodiode for identification of sentinel lymph node. In: $8^{\text {th }}$ International Conference on Inorganic Scintillators and their Use in Scientific and Industrial Applications, 2005, Crimea. Proceedings of Eight International Conference on Inorganic Scintillators and their Use in Scientific and Industrial Applications. Kharkov: National Academy of Sciences of Ukraine, 2005; 1:282-4.
11. Costa FE, Rela PR, Oliveira IB, Pereira MC, Hamada MM. Surgical gamma probe with $\mathrm{TIBr}$ semiconductor for identification of sentinel lymph node. IEEE Trans Nucl Sci. 2006; 53(3):1403-7.

12. Costa FE. Desenvolvimento de sondas cirúrgicas radioguiadas com semicondutores de $\mathrm{TIBr}$ e com cristais cintiladores de CSI (TI) [dissertação]. São Paulo: Instituto de Pesquisas Energéticas e Nucleares, USP; 2006.

13. Oliveira IB, Costa FE, Chubaci JFD, Hamada MM. Purification and preparation of $\mathrm{TIBr}$ crystals for room temperature radiation detector applications. IEEE Trans Nucl Sci. 2004; 51(3):1224-8.

14. Santos PCF, Santos IDAO, Nahas FX; Oliveira Filho RS, Ferreira LM. National equipment of intraoperatory gamma detection in the identification of sentinel lymph node in animal model. Acta Cir. Bras. 2009; 24(3):195-9.

Recebido em 13/01/2009

Aceito para publicação em 10/03/2009

Conflito de interesse: nenhum

Fonte de financiamento: nenhuma

\section{Como citar esse artigo:}

Filho RSO, Farah AB, Costa FE, Nahas FX, Oliveira DA, Oliveira RG. Equipamento brasileiro de detecção de gama intra-operatória para biópsia de linfonodo sentinela. Rev Col Bras Cir. [periódico na Internet] 2010; 37(1). Disponível em URL: http://www.scielo.br/rcbc

\section{Endereço para correspondência:}

Renato Santos de Oliveira Filho

E-mail: renato.prevencao@terra.com.br 